\title{
A Numerical Examination of the Performance of Small Magnetic Nozzle Thrusters
}

\author{
Timothy A. Collard, J. P. Sheehan†, and Benjamin A. Jorns ${ }^{\ddagger}$ \\ University of Michigan, Ann Arbor, MI, 48109, USA
}

\begin{abstract}
A modified version of the generalized performance model developed by Lafleur ${ }^{1}$ is used to interpret the low thrust performance measured for the CubeSat Ambipolar Thruster (CAT) in a previously reported ${ }^{2}$ thruster configuration. For partially magnetized ions the detachment criterion proposed by Lafleur, where detachment occurs when the local ion gyroradius equals the local plasma radius, is replaced with the turning point detachment criterion proposed by Little. ${ }^{3}$ The results from the original Lafleur model and the modified model are compared to indirect thrust measurements, and the plasma thrust predicted by the modified model matches the experimental data within errorbars. The modified model is used to investigate the change in performance due to variations in the design parameters and the operating conditions of the thruster. From this analysis the electron temperature is determined to be a critical parameter, and that at electron temperatures of $\lesssim 3 \mathrm{eV}$ marginal improvement of $\lesssim 2 \%$ in performance over cold gas operation is obtained. The primary mechanisms to control the electron temperature are shown to be the source aspect ratio $\left(R_{s} / L_{s}\right)$ and the propellant mass flow rate. To a lesser degree the detachment ion Mach number is shown to have an impact on the performance. The model also shows that the relatively low CAT thrust performance is due to a small aspect ratio of $\sim 0.12$ and a nominal operating mass flow rate that is an order of magnitude too high to get good performance $(\geq 500 \mu \mathbf{N})$. To improve the performance of CAT it is suggested that the aspect ratio be changed to $\sim 1$ and the operating propellant flow rate reduced to $\sim 25 \%$ of the current nominal flow rate. A discussion on how to maximize performance of generalized magnetic nozzle thrusters is included.
\end{abstract}

\section{Nomenclature}

$\beta \quad=$ Radial density average parameter, (-)

$\dot{m}_{C A T}=$ Nominal operating propellant flow rate of CAT, $(\mathrm{kg} / \mathrm{s})$

$\epsilon_{c} \quad=$ Collisional power loss, $(\mathrm{eV})$

$\epsilon_{e_{-}} \quad=$ Electron energy loss, $(\mathrm{eV})$

$\epsilon_{i-} \quad=$ Ion kinetic energy at the sheath edges, $(\mathrm{eV})$

$\eta_{m} \quad=$ Mass utilization efficiency, $(-)$

$\Gamma_{0} \quad=$ Particle number flux, $\left(/ \mathrm{m}^{2}-\mathrm{s}\right)$

$\mu_{0} \quad=$ Vacuum permeability, $(\mathrm{H} / \mathrm{m})$

$a_{m} \quad=$ Magnet radius, $(\mathrm{m})$

$B_{r} \quad=$ Radial component of the magnetic field, $(\mathrm{T})$

$B_{z} \quad=$ Axial component of the magnetic field, (T)

$h_{R} \quad=$ Sheath edge-to-center density ratio, (-)

$I=$ Current through the loop to generate the magnetic field, (A)

$K_{\text {exc }}=$ Excitation rate constant, $\left(\mathrm{m}^{-3} / \mathrm{s}\right)$

$K_{i z} \quad=$ Ionization rate constant, $\left(\mathrm{m}^{-3} / \mathrm{s}\right)$

$L_{s} \quad=$ Source length, $(\mathrm{m})$

*Ph.D. Candidate, Department of Aerospace Engineering, University of Michigan, and AIAA Student Member.

$\dagger^{\dagger}$ Mission Systems Engineer, OneWeb, and AIAA Member.

${ }^{\ddagger}$ Assistant Professor, Department of Aerospace Engineering, University of Michigan, and Senior AIAA Member. 




\section{Introduction}

D UE to reduced cost, rapid development timetables, and increased capabilities of miniaturized sensors, CubeSats have become attractive options to both commercial and scientific groups interested in a wide range of mission types. These missions include flexible communications constellations, long-lived, low altitude flights to monitor weather and agricultural conditions, and health monitoring of other on-orbit assets. Many of these applications require satellite maneuverability exceeding the capabilities of current state-ofthe-art chemical and electric thrusters ${ }^{4-25}$ The CubeSat Ambipolar Thruster (CAT) ${ }^{26}$ is an electrodeless, inductively-coupled magnetic nozzle thruster that ionizes propellant using radio frequency (RF) power. By operating without a plasma-wetted electrode and by confining the plasma away from the walls through shaped magnetic fields thruster wear is minimized and reactive propellants can be used. The $50 \mathrm{~W}$ unit built at the Plasmadynamics and Electric Propulsion Laboratory (PEPL) at the University of Michigan was designed to meet the stringent maneuverability requirements of many missions of interest. In this device the plasma expands through a diverging magnetic nozzle that converts the thermal energy contained within the plasma into directed kinetic energy. However, based on indirect thrust measurements of CAT in the reported configuration, ${ }^{2}$ it is unclear that the system offers improved maneuverability.

Similar magnetic nozzle devices of varying size and power have also demonstrated poor performance, ${ }^{3,27-31}$ often with total efficiencies less than $5 \%$ and only modest thrust gains over cold gas systems. The questions then arise: 1) Can a model be developed to estimate the performance of CAT, while being consistent with measured performance?, 2) Is poor performance inherent to these devices?, and 3) Can the validated model identify changes to improve the performance of CAT and other magnetic nozzle thrusters? Previous models of RF thrusters, constructed Fruchtman, et. al. ${ }^{32}$ and Lafleur, ${ }^{1}$ that were able to estimate performance of up to $2 \mathrm{~kW}$ thrusters with fidelity. ${ }^{33}$ However, the CAT is not necessarily captured by these models and poses a unique challenge because of its size, low-power requirements, and partially magnetized ions. Modification of these models, which are designed for larger thrusters with magnetized ions, is required to make them applicable to small thrusters, such as the CAT, to allow for prediction of performance and to understand what drives the performance. In this work the ion detachment criterion is modified and the predicted performance is compared to experimental measurements to provide a predictive tool to the electric propulsion community. In Section II the generalized model developed by Lafleur ${ }^{1}$ to estimate thruster performance will be leveraged with a modification to the detachment criterion to answer the first question. To address the remaining two questions, the results of the model are compared to indirect thrust measurements of the CAT in Section IV then a series of parameters within the model are varied. The results of this parameter investigation are presented in Section V, allowing a critical parameter to be identified and prompting a discussion how devices, such as CAT, can incorporate this information to improve thrust performance.

\section{Theoretical Model}

The theoretical model used in this work is based on the quasi-1D formulation developed by Lafleur. ${ }^{1,34}$ For completeness and discussion the primary components of said model are summarized in this section. The model is equipped to handle a generalized RF magnetic nozzle thruster. In this thruster configuration, an $\mathrm{RF}$ antenna couples power to free electrons within a source tube that is injected with neutral propellant to ionize the gas. The plasma then expands through a diverging magnetic nozzle imposed by an externally applied magnetic field. At some point in the diverging magnetic nozzle section, the plasma demagnetizes from the field lines and no longer transmits force upstream to the thruster through the magnetic nozzle. Note 
that a key assumption within this model is that the ions diverge more slowly than the magnetic field lines beyond the detachment point. As noted by Lafleur, there are works that suggest that ions may diverge more rapidly than the field lines under certain conditions. ${ }^{35-38}$ Figure 1 depicts a general magnetic nozzle thruster and clarifies dimensions and terminology contained within this section.

The first step in Lafleur's model is to solve the momentum and continuity equations within the source region. To reduce the problem to one dimension while accounting for the radial density variations within the source tube a self-similar profile derived empirically is assumed of the form

$$
f(\rho)=\left[1-\left(1-h_{R}^{1 / t}\right) \rho^{s}\right]^{t}
$$

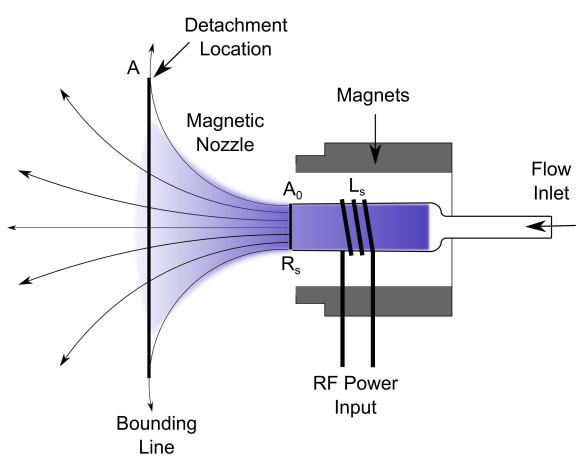

Figure 1: A notional diagram of a magnetic nozzle thruster. Note the clarifying definition of the source dimensions and areas used in the model.

where $\rho=r / R_{s}$. Based on fits to experimental data the parameters are $t=6$ and $s=2$. A radial average of the density can be determined from $\langle n f\rangle=\beta n$, where $\beta$ is defined as

$$
\beta=2 \int_{0}^{1}\left[1-\left(1-h_{R}^{1 / t}\right) \rho^{s}\right]^{t} \rho d \rho
$$

Through manipulation of the ion and electron momentum equations within the source region, accounting for the axial variations and radial averaging, and assuming a collisionless plasma and minimal momentum loss to the walls and a constant cross-sectional area this yields the momentum balance

$$
-2-\xi \ln \left(\frac{1-\xi}{1+\xi}\right)+2 \sqrt{1-\xi^{2}}\left[\tan ^{-1}\left(\frac{1-\xi}{\sqrt{1-\xi^{2}}}\right)-\tan ^{-1}\left(\frac{-1-\xi}{\sqrt{1-\xi^{2}}}\right)\right]=\gamma
$$

with the various grouped terms defined as

$$
\begin{gathered}
\gamma=a \frac{K_{i z}}{u_{B}}-b, \\
\xi=\frac{1}{1-\frac{u_{B} b}{K_{i z}} \frac{}{a}}, \\
a=\frac{\Gamma_{0} L_{s}}{v_{g}},
\end{gathered}
$$

and

$$
b=\frac{2 h_{R} L_{s}}{\beta R_{s}}
$$

Note that the ionization rate, $K_{i z}\left(T_{e}\right)$, and the Bohm speed, $u_{B}\left(T_{e}\right)$ are functions of electron temperature while the particle flux, $\Gamma_{0}\left(\eta_{m}\right)$, is a function of the mass utilization efficiency. This result is a function of the thruster design (geometry), operating point (propellant flow rate), and a free parameter (electron temperature). Eq. 6 is a function of the thruster operating conditions and the residence time of the propellant within the source while Eq. 7 is a function of the thruster source region aspect ratio. Physically, this demonstrates that there is an equilibrium electron temperature required to balance the losses to the wall and out of the thruster with the ionization within the source. In practice, once the thruster design is determined and an operating condition is selected Eq. 3 can be iterated to determine the electron temperature. Note 
that within this analysis the electron inertia and radial ion losses are neglected, and the ions are assumed to be cold.

In the diverging nozzle section the electron and ion momentum equations can be combined with the continuity equation to yield a relationship between the expansion of the nozzle and the ion acceleration, of the form

$$
\frac{1}{2}\left(M_{\text {det }}^{2}-1\right)-\ln \left(M_{\text {det }}\right)=\ln \left(\frac{A}{A_{0}}\right)
$$

This equation holds until the detachment point, beyond which the ions no longer follow the imposed field lines. The plasma within this expanding section is assumed to be collisionless and perfectly confined by the bounding magnetic field line originating at the walls at the source exit plane.

In the Lafleur model the ions are assumed to detach when the local ion gyroradius exceeds the local plasma radius. This asserts that the ions are magnetized when leaving the source, or that the ion Larmor radius is much smaller than the source diameter. This assumption does not hold for CAT due to its small source diameter, such that $R_{c i} / R_{s} \geq 1$. This suggest that the Lafleur criterion is not appropriate for small thrusters so an alternative criterion proposed by Little ${ }^{3}$ will be introduced at the end of this section.

In order to predict efficiency performance a total power must be estimated. Within the source region the power absorbed by the plasma is equal to the power loss at the boundaries,

$$
P_{a b s}=\frac{1}{2} \beta n_{0} u_{B} A_{0}\left(\epsilon_{c}+\epsilon_{i c}+\epsilon_{e c}\right)+\frac{1}{2} \beta n_{0} u_{B} A_{0}\left(\epsilon_{c}+\epsilon_{i 0}+\epsilon_{e 0}\right)+\frac{\pi q u_{B} R_{s} h_{R} n_{0} L_{s}}{\gamma \xi} \ln \left(\frac{1+\xi}{1-\xi}\right)\left(\epsilon_{c}+\epsilon_{i r}+\epsilon_{r}\right)
$$

where the first term is the power loss at the back wall, the second term is at the exit plane, and the last term is the radial losses. The various energy loss terms are defined as

$$
\begin{gathered}
\epsilon_{c}=\epsilon_{i o n}+\frac{K_{e x c}}{K_{i z}} \epsilon_{e x c} \\
\epsilon_{e c}=\epsilon_{e r}=2 T_{e}+\frac{T_{e}}{2} \ln \left(\frac{m_{i}}{2 \pi m_{e}}\right), \\
\epsilon_{e 0}=\frac{1}{2} M_{d e t}^{2} T_{e}+2 T_{e}
\end{gathered}
$$

and

$$
\epsilon_{i c}=\epsilon_{i 0}=\epsilon_{i r}=\frac{T_{e}}{2}
$$

and capture losses due to collisions, the sheath inside the source, and electron transport at the boundaries of the source region. The third term in Eq. 9 physically captures wasted power absorbed by the radial walls of the source, and the dependence on the thruster geometry suggests that it can be minimized through careful design of the source aspect ratio.

Turning to the magnetic nozzle section, the additional thrust produced by the nozzle can be determined from combining the ion and electron momentum equations and integrating over the cross sectional area at the detachment point, resulting in

$$
F_{P}=\left(\frac{M_{d e t}^{2}+1}{2 M_{\text {det }}}\right) F_{0}
$$

where $F_{0}$ can be interpreted as the upstream thrust:

$$
F_{0}=q \beta n_{0} T_{e} A_{0}
$$

As noted above, this model assumes magnetized ions to determine the detachment point, but this does not hold for CAT due to its small size. An alternative criterion, proposed by Little based on the results of Merino and Ahedo, ${ }^{3,39}$ is to assume that plasma remains confined by the nozzle up to the turning point of the 
bounding field line. Beyond the turning point of the bounding field line minimal momentum is transferred to the thruster, so, for purposes of estimating thrust performance, detachment can be assumed to occur at the turning point. To find the turning point the exact cylindrical solution for the magnetic field topology generated by a current loop, ${ }^{40}$

$$
\begin{gathered}
B_{r}=\frac{\mu_{0} I z}{2 \pi \alpha_{m}^{2} \beta_{m} r}\left[\left(a_{m}^{2}+r^{2}+z^{2}\right) E\left(k^{2}\right)-\alpha_{m}^{2} K\left(k^{2}\right)\right], \\
B_{z}=\frac{\mu_{0} I}{2 \pi \alpha_{m}^{2} \beta_{m}}\left[\left(a_{m}^{2}-r^{2}-z^{2}\right) E\left(k^{2}\right)+\alpha_{m}^{2} K\left(k^{2}\right),\right] \\
\alpha_{m}^{2}=a_{m}^{2}+r^{2}+z^{2}-2 a_{m} r \\
\beta_{m}^{2}=a_{m}^{2}+r^{2}+z^{2}+2 a_{m} r
\end{gathered}
$$

and

$$
k^{2}=1-\frac{\alpha_{m}^{2}}{\beta_{m}^{2}} .
$$

The turning point can be determined by iteratively solving for the point along the bounding field line at which $B_{z}=0$. For clarity, the turning point is the red dot in Figure 2.

The cross sectional area of the nozzle at the detachment point then becomes $A=\pi r_{t p}^{2}$, allowing the Mach number of the ions at the detachment point to be determined from Eq. 8. The rest of the Lafleur algorithm remains unchanged; a mass utilization efficiency can be chosen, and Eqs. 3, 8, and 9 can be iteratively solved for the electron temperature, detachment Mach number, and absorbed power, respectively. Then the thrust, specific impulse, and total efficiency can be determined. This algorithm is summarized in the flow chart in Figure 3.

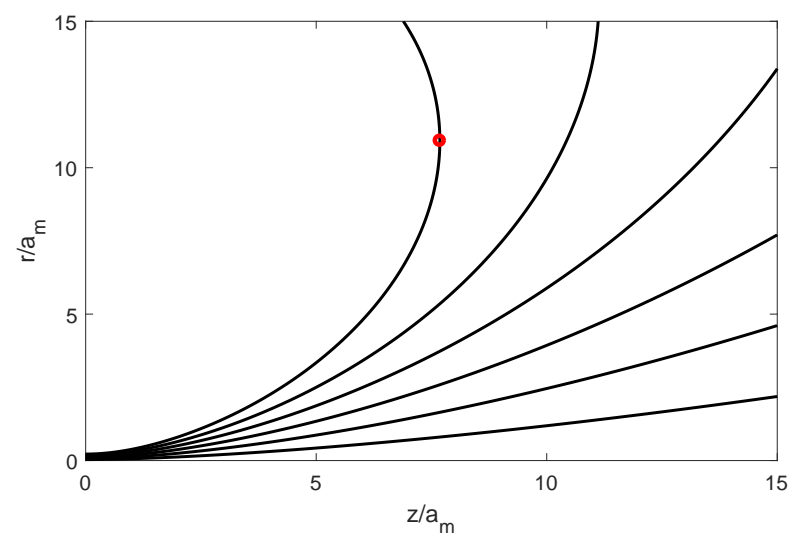

Figure 2: The location of the turning point for a diverging magnetic nozzle.

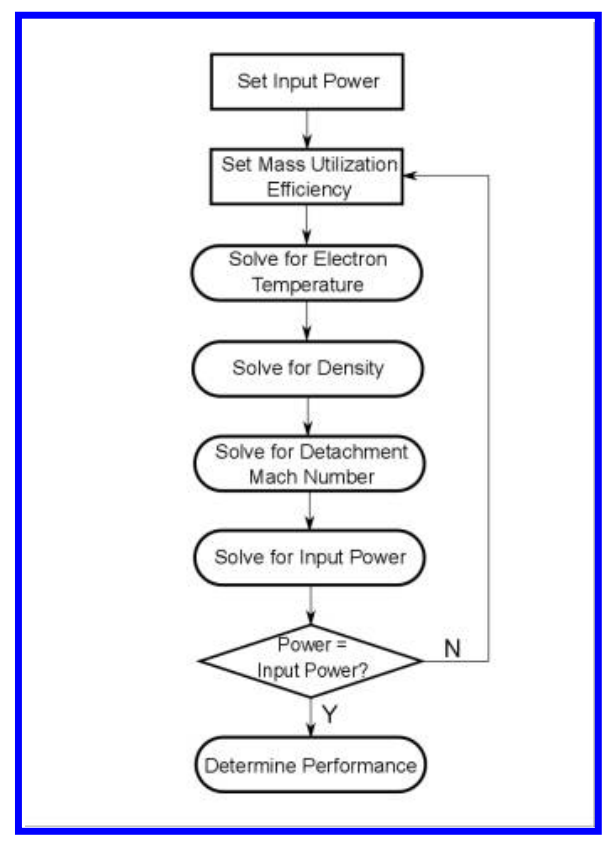

Figure 3: An outline of the algorithm used to predict thruster performance. 


\section{Experimental Setup}

To compare the theoretical performance to experimental data the thrust is indirectly measured in the Junior Test Facility connected to the Large Vacuum Test Facility at the University of Michigan. Junior is a 3 meter long, by 1 meter diameter stainless steel clad vacuum chamber capable of achieving base pressures of $3 \times 10^{-6}$ Torr and a background pressure of $5 \times 10^{-4}$ Torr during thruster operation at propellant flow rates up to $3.5 \mathrm{mg} / \mathrm{s}$. The chamber was maintained at high vacuum by a turbopump with a nominal pumping speed of $1650 \mathrm{~L} / \mathrm{s}$ on nitrogen or $800 \mathrm{~L} / \mathrm{s}$ on xenon. As depicted in Figure 4 a momentum target affixed to the null-type torsional thrust stand described by Collard and Sheehan ${ }^{2}$ is used to indirectly measure the thrust. During this experiment the CAT is configured as described by Collard and Sheehan. ${ }^{2}$

The CAT, in Figure 5, is an electrodeless, inductively-coupled magnetic nozzle thruster that converts thermal energy within the plasma into thrust. This unit has an onboard power processing unit (PPU) that converts DC power to RF power at the thruster antenna. The mesh in Figure 5 is used to protect the PPU from secondary plasma discharges due to the high background pressures of $5 \times 10^{-4}$ Torr in the experiment. The CAT is operated at $9.929 \mathrm{MHz}$ and at up to $50 \mathrm{~W}$ input power. To limit maximum operating temperature the system is backed by an open-loop water cooling system that rejects waste heat outside the vacuum chamber. To precisely control the operating parameters of the thruster laboratory power supplies and an external propellant feed system is employed.

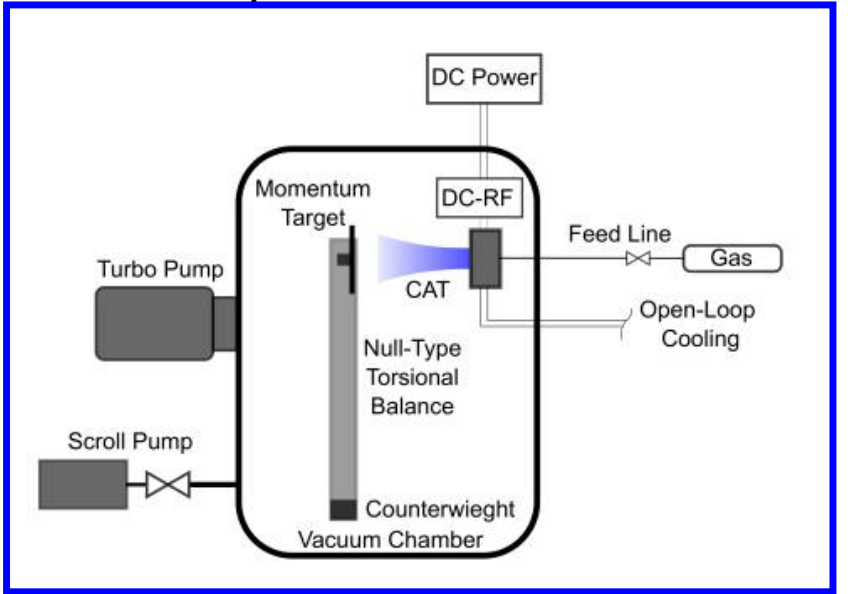

Figure 4: The setup used to indirectly measure thrust from CAT.



Figure 5: The integrated CAT unit that was performance tested.

\section{Results}

In this section experimental indirect thrust measurements of the CAT will be presented. The models, using both the magnetized and partially magnetized ion criteria, will be compared to this thrust data to determine if they are useful predictive tools. To accurately predict the CAT performance empirical parameters used in the original Lafleur model ${ }^{1}$ are modified to better match the nominal CAT configuration.

\section{A. Thrust}

The model outlined in Section II is developed to predict thruster performance, however until it is compared to experimental data it remains a theory. The CAT thrust data has been previously indirectly measured, ${ }^{2}$ but the reported values did not fully account for error-inducing effects present in the experiment, such as thrust stand hysteresis and RF pickup. To account for these effects another experimental campaign was conducted to determine the thrust performance. This results in a revised indirect thrust measurements in Figure 6 , down from the mid 10 s of $\mu \mathrm{N}$ of thrust generated by the plasma. During this experiment the thrust was operated in a consistent pattern; off - cold gas - plasma firing - cold gas - off; to capture the hysteresis of the thrust stand. The artificial thrust due to RF pickup on the thrust stand was measured by recording the cold gas thrust and then turning the RF power on without creating a plasma. Additionally, the thrust stand has not been validated, so additional error is included in the errorbars on the thrust reported in Figure 6 . 


\section{B. Density}

To fully adapt the model in Section II to the CAT configuration the density measurements of a device similar to CAT that is under development at PEPL is fit to capture the radial plasma variation within the source region. These density measurements were made using a double Langmuir probe swept across the face of the thruster at the exit plane. As shown in Figure 7 the density measurements are well fit when the fitting parameters are $t=-794.5$ and $s=3.2$. This results in a revised $\beta$ of 0.72 .

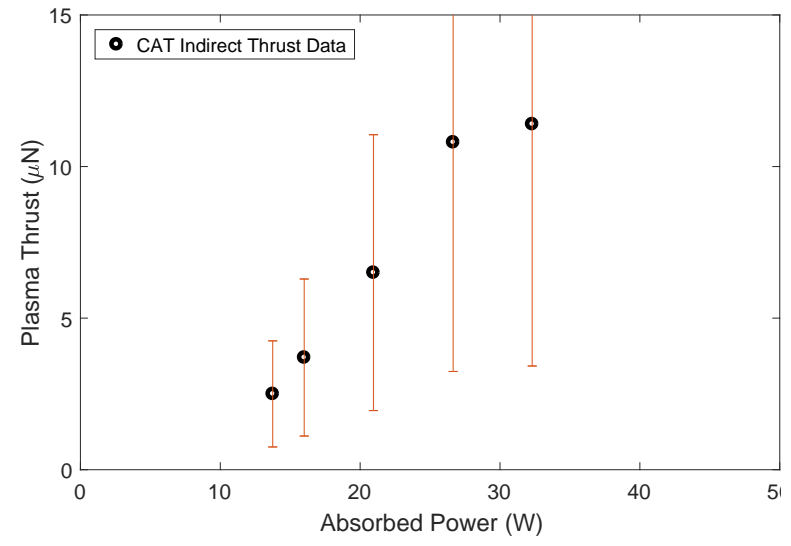

Figure 6: The indirectly measured thrust generated by the CAT. The errorbars include hysteresis of the thrust stand, RF pickup on the measurement circuit, and that the thrust stand is not validated.

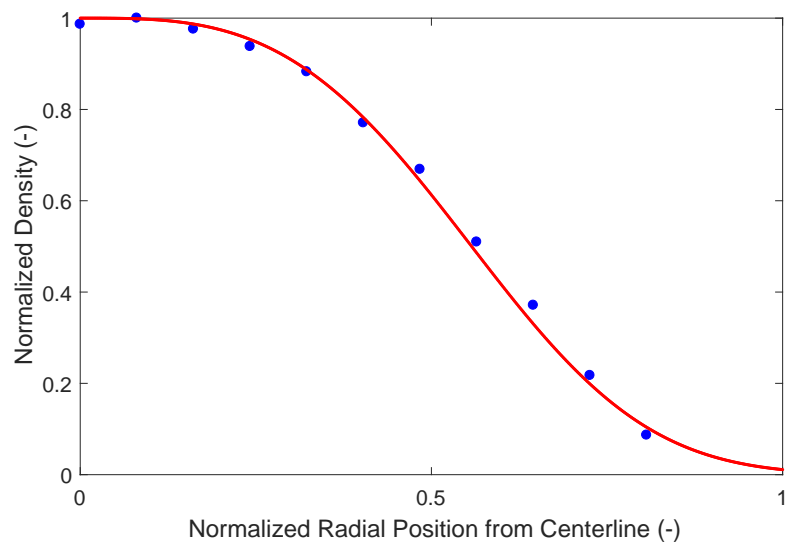

Figure 7: The fit (line) to experimental density data (dots) at the throat of a magnetic nozzle source similar to CAT under development at PEPL. The best fit parameters are $t=-794.5$ and $s=3.2$.

\section{Comparison of Models and Measurements}

By including the measured density variations and incorporating the thruster design and operating point the models can be compared to the indirect thrust measurements. As observed in Figure 8 the original Lafleur model does not match the thrust measurements well. This is unsurprising because magnetized ions are assumed, while the ions in CAT are partially magnetized. However, when the detachment criterion is modified to account for partially magnetized ions the model fits the indirect thrust measurements within the errorbars of the measurements.

Since the model fits the experimental data the model can provide insight into why the thrust performance is low. As shown in Figure $9 \sim 86 \%$ of the power deposited into the source region is lost to the radial walls, while only $\sim 7 \%$ of the power contributes to thrust generation. The natural question that then arises is which design parameters are critical to performance and what level of thrust performance can be obtained in these devices? The good agreement between the modified model and the indirect thrust measurements suggests that the



Figure 8: The thrust generated by the magnetic nozzle,assuming magnetized ions, and the model with the modified detachment criterion. The model with the modified criterion converges to a $T_{e}=2.7 \mathrm{eV}$ and the estimated plasma thrust performance of the modified model fits the indirect thrust measurements from CAT, within the experimental errorbars. model accounts for the primary physics driving performance of small magnetic nozzle thruster and can be used to explore this question. In the next section the nominal CAT configuration is used as the baseline performance case and the impact of design parameters on thrust performance is examined by varying an individual parameter throughout the range applicable to a CubeSat. 


\section{Discussion}

The modified model can be used to identify the critical parameters impacting thrust performance by varying design parameters. Once the critical parameters are identified the design of small magnetic nozzle thrusters can be optimized. This section will explore the parameter space and comment on the resulting design impacts.

\section{A. Performance Impact of Parameter Variation}

To explore the available thrust performance space the model can be accept a nominal thruster design and operating point as the baseline. The model can then explore the performance impact of varying a single parameter from its nominal value. For the CAT the three controllable parameters explored are the detachment point via changes to the magnetic nozzle topology, the propellant flow rate, and the source aspect ratio. The magnetic field strength is not included because the CAT already uses the strongest manufacturable permanent magnets. The resulting thrust performance predicted by the model is discussed below for each of these cases.

\section{Detachment Point}

The first parameter varied is the detachment point via varying the radius of the magnet, $a_{m}$. As detailed in Figure 2, the detachment location scales

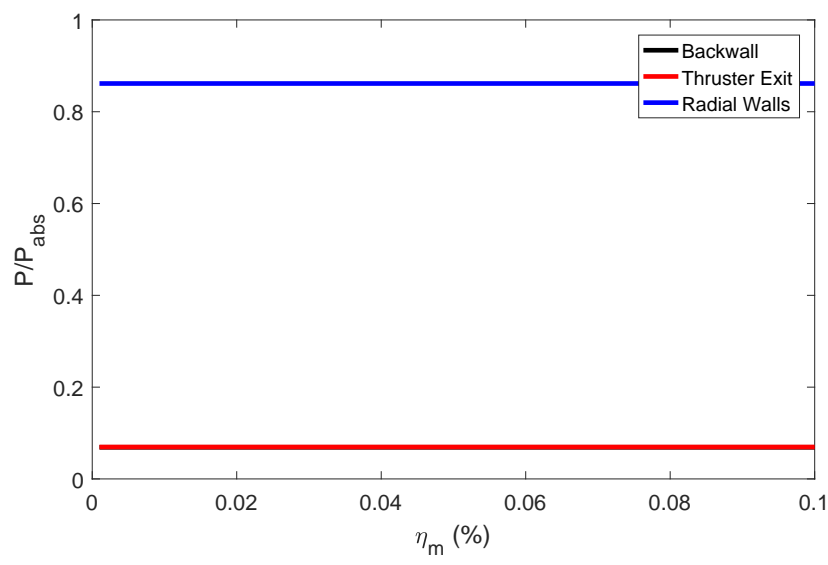

Figure 9: The power loss to the three source boundaries for the nominal CAT configuration. Note that the thruster exit and the backwall overlap. with the magnet radius, therefore the detachment radius is a function of the magnet radius. Changes in the detachment radius impacts the expansion ratio in Eq. 8, which dictates the detachment Mach number and resulting thrust performance. To evaluate the impact of detachment point on performance the magnet radius is doubled and halved, while all other thruster design and operating conditions are held at the nominal values.

As shown by Figure 10 the changes in the thrust are $\sim 10 \%$, suggesting that the magnetic field topology can improve performance but it not a critical parameter. The contribution of the plasma to the total thrust is still low, at $\sim 1 \%$ of the cold gas thrust. There are only negligible changes to the predicted specific impulse and total efficiency, and both remain low. This lends credence to the assertion that the detachment point is not a primary driver of performance.

\section{Propellant Flow Rate}

The magnet radius is then returned to the nominal design condition and the propellant flow rate is varied. Variations in the propellant flow rate change the particle flux embedded within the source continuity equations that determine the electron temperature. The electron temperature is also the critical controllable parameter in the upstream plasma thrust in Eq. 15. To determine the impact of propellant flow rate on the electron temperature and the overall thrust performance the flow rate is incrementally decreased from the nominal value to a tenth of the nominal value.

As observed in Figure 11a the electron temperature nonlinearly increases with decreasing propellant flow rate at a fixed input power of $50 \mathrm{~W}$. Fundamentally, this is stating that as the neutral density within the source region decreases the the electron temperature must increase to maintain the absorbed power. Due to the scaling of the upstream thrust with electron temperature this is a direct way to improve thrust performance. As shown in Figure 12 a decrease in flow rate to $10 \%$ of the nominal operating condition results in a thrust increase of $\sim 100 \%$ at $50 \mathrm{~W}$, suggesting that this is a critical parameter impacting thrust performance. At sufficiently high electron temperatures, $T_{e} \geq 5 \mathrm{eV}$, the magnetic nozzle contribution to total thrust approaches the level generated by the cold gas neutral flow within the $50 \mathrm{~W}$ power range. This is accompanied by a modest increase in the specific impulse of the device due to an increase in the effective exhaust velocity of the plasma. However, the total efficiency drops with decreasing propellant flow rate due 


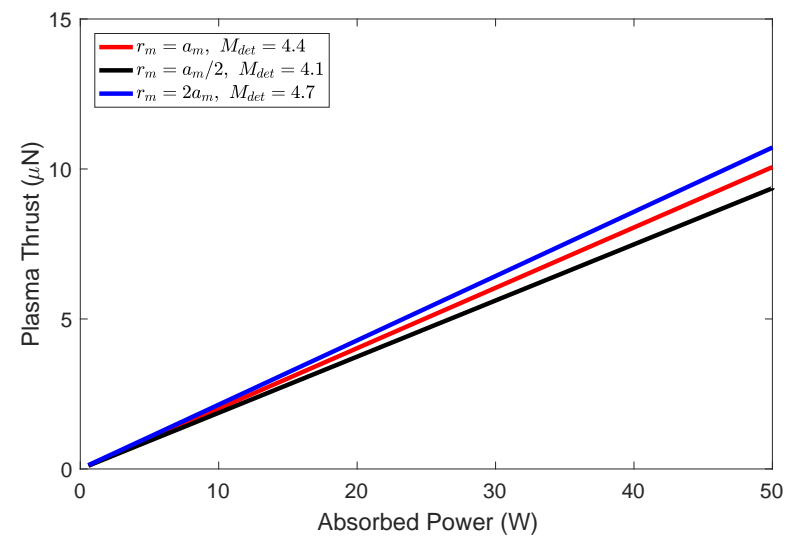

$((\mathrm{a}))$



$((\mathrm{c}))$

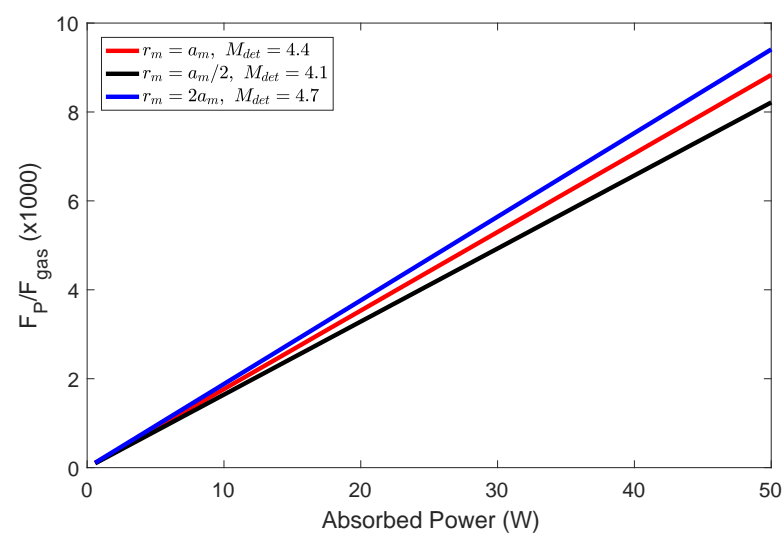

((h))

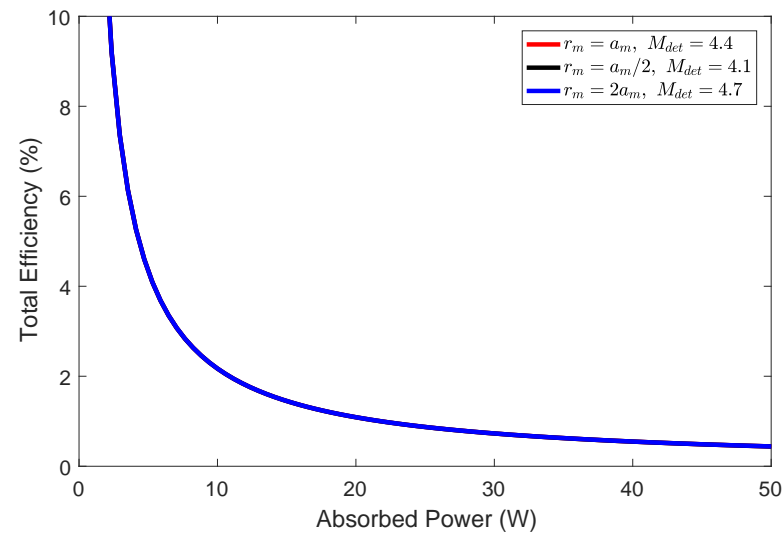

$((\mathrm{d}))$

Figure 10: The change in (a) plasma thrust, (b) the ratio of the plasma thrust and cold gas thrust, (c) the specific impulse, and (d) total efficiency performance with variations in the detachment Mach number. The detachment Mach number was changed by varying the nozzle expansion ratio, which is a function of the magnet radius. Note that the total efficiencies in (d) overlap for all three cases.

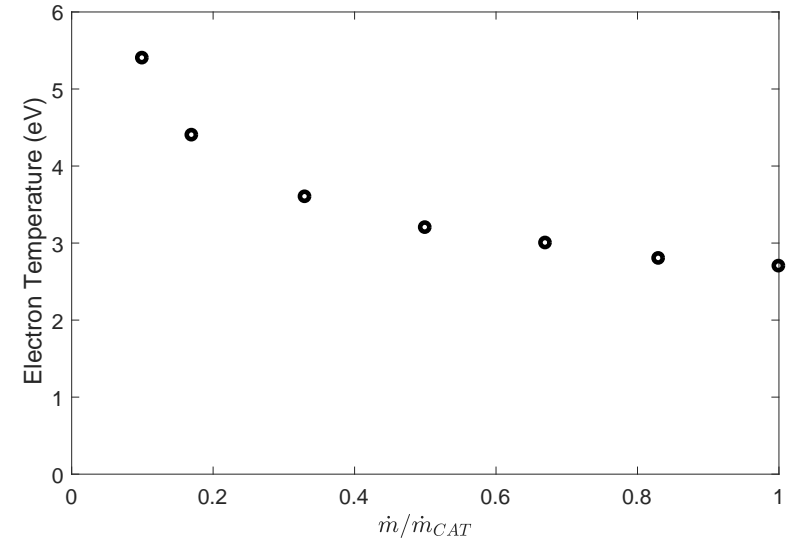

(a)

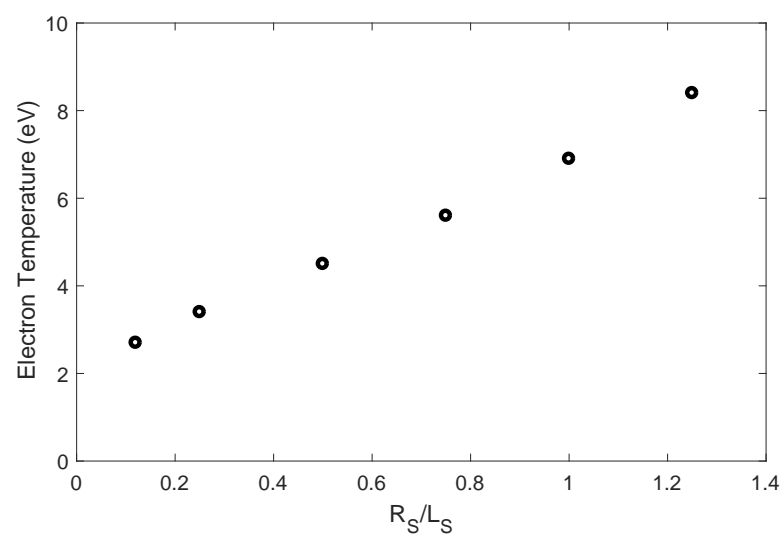

(b)

Figure 11: (a) The relationship between input propellant flow rate and electron temperature. (b) The increase in electron temperature with increasing source aspect ratio. Note that the nominal CAT configurations is the far left point in (b) with an $R_{s} / L_{s}=0.12$. 
to the smaller total thrust being generated. This is largely due to the fact that the cold gas thrust remains a non-negligible thrust term. Despite the low efficiency performance the improvements in the contribution of the plasma to thrust and the specific impulse also suggest that propellant flow rate is an important performance parameter.

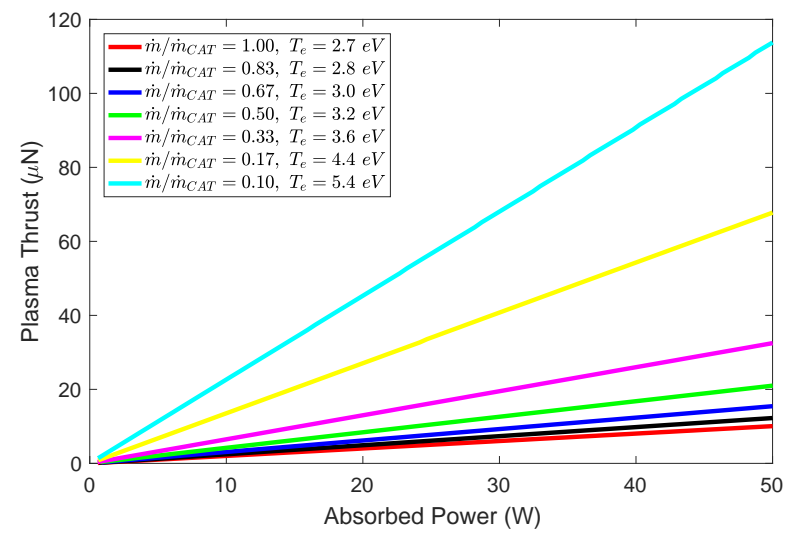

$((\mathrm{a}))$

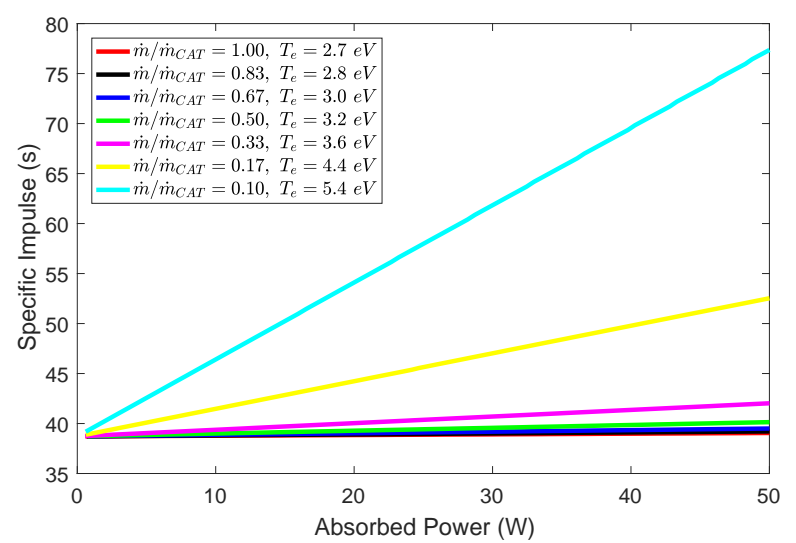

$((\mathrm{c}))$



((b))

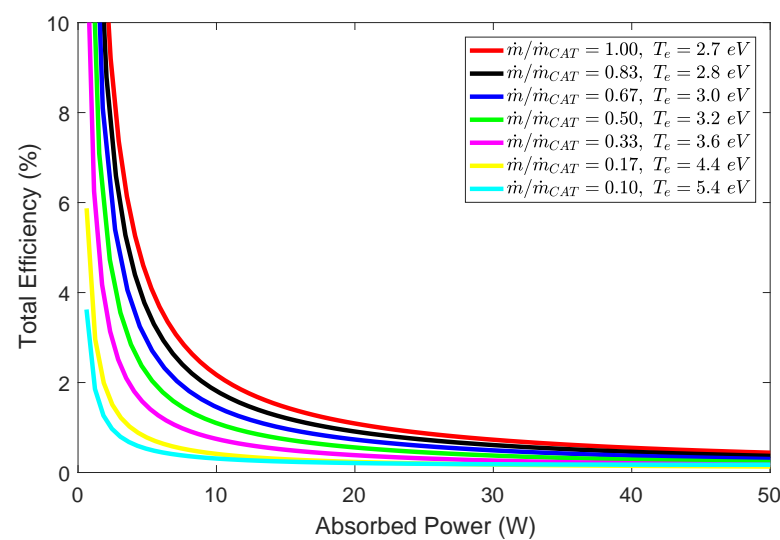

$((\mathrm{d}))$

Figure 12: The change in (a) plasma thrust, (b) the ratio of the plasma thrust and cold gas thrust, (c) the specific impulse, and (d) total efficiency performance with decreasing propellant flow rate. Note that $T_{e}=2.7 \mathrm{eV}$ is the electron temperature of the nominal CAT configuration.

\section{Source Aspect Ratio}

Finally, the propellant flow is returned to the nominal value and the source aspect ratio is varied. Changes in the source aspect ratio impact the energy equation within the source region by reducing the radial wall losses and the source momentum equation by changing the electron temperature required to balance the power absorbed and the particle flux through the source. To quantify the impact of source aspect ratio on the performance the aspect ratio is incrementally increased.

As shown in Figure $11 \mathrm{~b}$ the electron temperature increases linearly with increasing aspect ratio. Due to the scaling of upstream thrust with the electron temperature this is another way to directly control performance. An increase in the source aspect ratio by a factor of $\sim 10$ results in an $\sim 700 \%$ increase in the plasma thrust at $50 \mathrm{~W}$, as shown in Figure 13, accompanied by a $3 \times$ increase in electron temperature. This suggests that the aspect ratio is also a design parameter critical to performance. Like the decrease of propellant flow rate and increase in the aspect ratio results in a larger plasma component of the total plasma component. When compared to the impact of propellant flow rate on the relative thrust components, in Figure 12 , the aspect ratio appears to have a smaller impact. However, when the propellant flow rate is decreased the cold gas thrust contribution decreases, enhancing the percentage of total thrust contributed by 
the plasma. Since the suboptimal, nominal propellant flow rate is used within this case to examine the effect on performance of only the aspect ratio, the cold gas component of thrust is high compared to the higher performing $10 \%$ flow rate case of the previous section. This implies that the raw plasma thrust increase due to increasing the aspect ratio is actually higher than the improvement in reducing propellant flow rate. Therefore, a thruster with optimized performance will maximize the source aspect ratio and minimize the propellant flow rate. Like the propellant flow rate an increase in aspect ratio results in a modest increase in specific impulse. The total efficiency remains low, but increasing aspect ratio yields marginal gains due to the increase in thrust at a fixed power and propellant flow rate. Despite the low efficiency performance the aspect ratio is clearly a critical performance parameter.

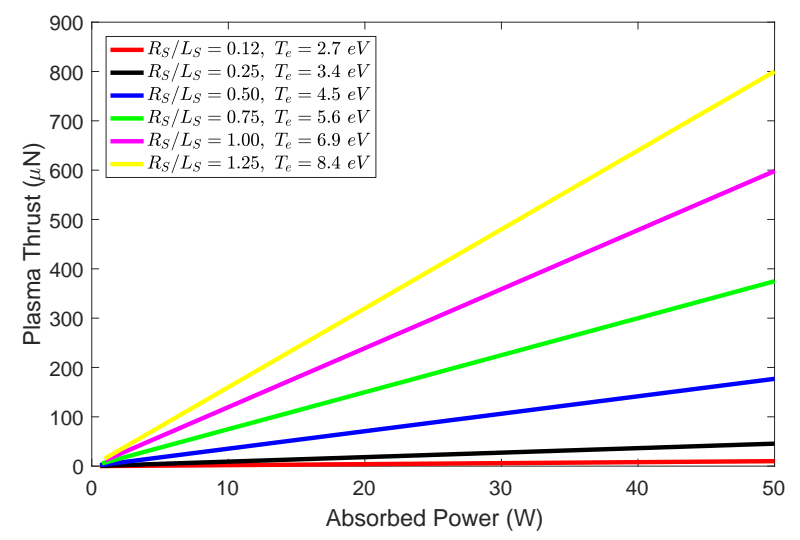

$((\mathrm{a}))$

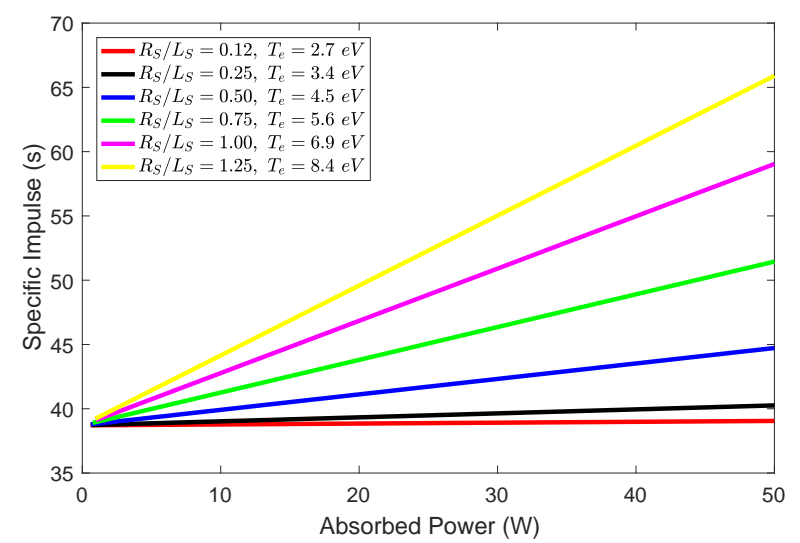

$((\mathrm{c}))$

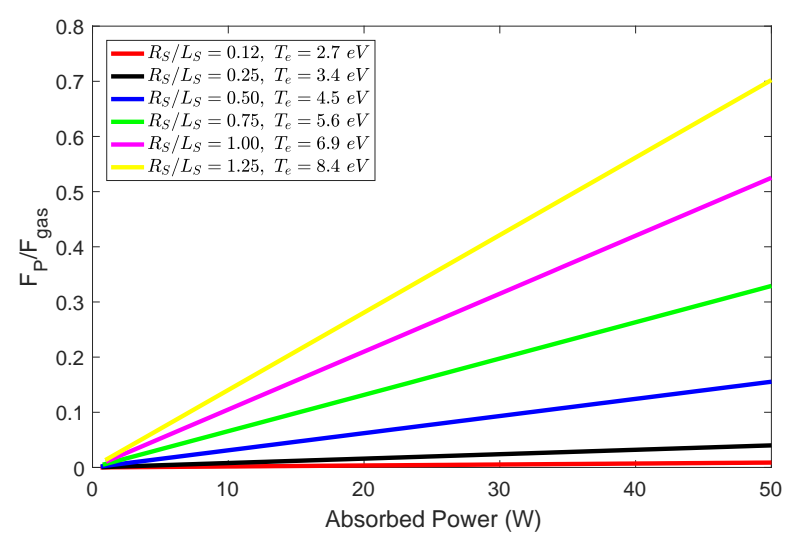

((b))

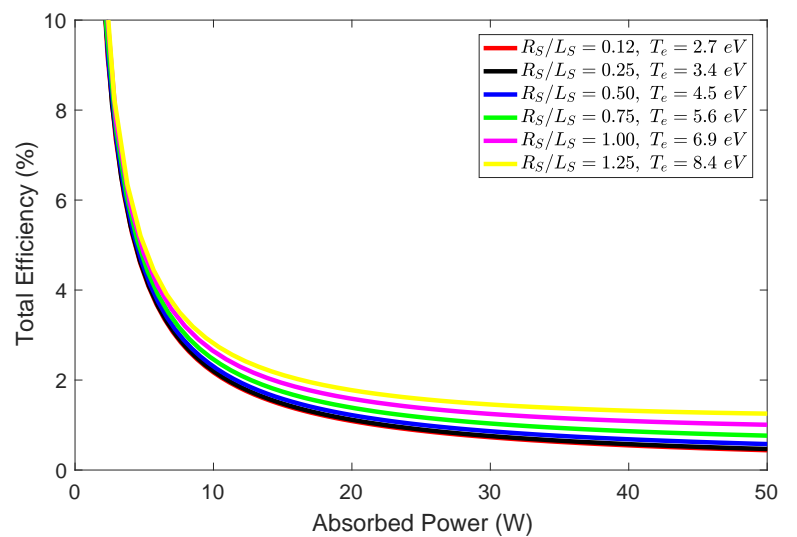

$((\mathrm{d}))$

Figure 13: The change in (a) plasma thrust, (b) the ratio of the plasma thrust and cold gas thrust, (c) the specific impulse, and (d) total efficiency performance with increasing source aspect ratio. Note that $T_{e}=2.7$ $\mathrm{eV}$ is the electron temperature of the nominal CAT configuration.

\section{B. Design Recommendations and Corresponding Performance}

As demonstrated in the previous subsection the aspect ratio and the propellant flow rate are critical parameters influencing performance of small magnetic nozzle thrusters. The design and nominal operating conditions of the the CAT are suboptimal for both of these parameters. The natural question that arises is what should the CAT configuration be to maximize performance?

Ideally, the aspect ratio should be maximized and the propellant flow rate minimized to maximize the performance. The constraints that define these optimal points are derived from the volume, mass, and power budget allocated for the thruster and the requirement of steady operation. In the case of the CAT, the thruster is nominally designed to be packaged within a $10 \mathrm{~cm}$ cube and to operate at up to $50 \mathrm{~W}$ input power. The physical size constrains the aspect ratio of the source, both in the length and diameter of the 
source liner. The magnetic circuit to impose the diverging magnetic nozzle must be included within this size constraint, resulting in a target CAT source aspect ratio of $\sim 1$. The propellant flow rate target is limited by the requirement of steady operation. If the flow rate is lowered below a certain threshold the thruster will operate unsteadily due to neutral depletion and inconsistent discharges. For the CAT, based on this consideration, the propellant flow rate target is $\sim 33 \%$ of the nominal flow rate.



$((\mathrm{a}))$



$((\mathrm{c}))$

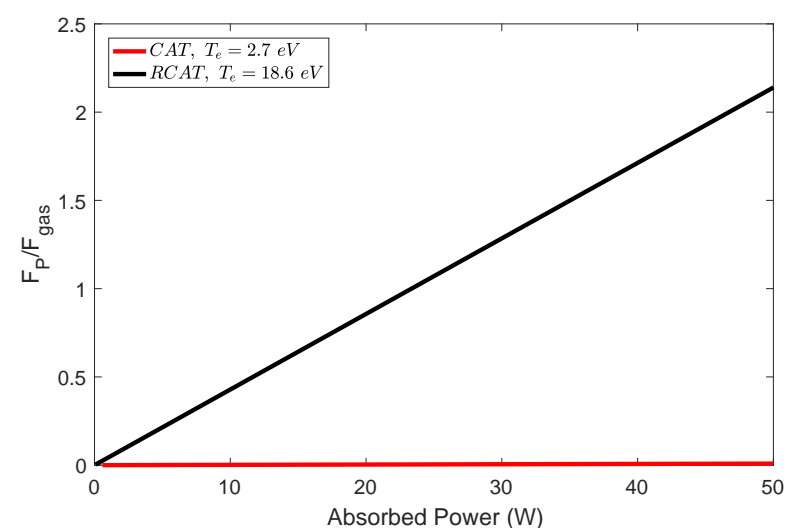

$((\mathrm{b}))$



$((\mathrm{d}))$

Figure 15: The change in (a) plasma thrust, (b) the ratio of the plasma thrust and cold gas thrust, (c) the specific impulse, and (d) total efficiency performance of the recommended CAT configuration compared to the nominal CAT.

The model can readily accept these two changes and report predicted performance across the $50 \mathrm{~W}$ operating range of the CAT. The revised CAT performance incorporates only these two parameter changes; the magnetic circuit and all other conditions remain the nominal CAT values. As shown in Figure 15, the performance of the revised CAT exhibits significant thrust performance compared to the nominal CAT configuration. In the revised configuration the thrust is $\sim 700 \%$ higher than the nominal configuration, and the plasma contribution to the total thrust reaches the order of the cold gas component at $\sim 25 \mathrm{~W}$. At power levels above $25 \mathrm{~W}$ the plasma thrust contributes more to the total thrust than the cold gas, suggesting that the magnetic nozzle device is starting to provide significantly improved performance over a cold gas system operating at the same propellant flow rates. This improved thrust performance is accompanied by modest gains in both the specific impulse and the total thruster efficiency. An examination of the energy balance within the source explains these performance improvements, As observed in Figure 14, in the revised configuration the power lost to the radial walls is reduced to $40 \%$ of the input power, compared to $86 \%$ in the nominal configuration. Therefore, significantly more power is contributing to thrust generation. Note that the model is blind to power deposition mechanism, so an electron temperature of $18.6 \mathrm{eV}$ may not be attainable for the original CAT inductively coupled design. To attain this high electron temperature the power coupling mechanism may need to be modified. Despite these performance gains, both the specific 
impulse and the efficiency remain low, prompting the question: is the low performance a function of poor performance scaling with power, or is it fundamentally limited by the physics underlying the operation of these devices?

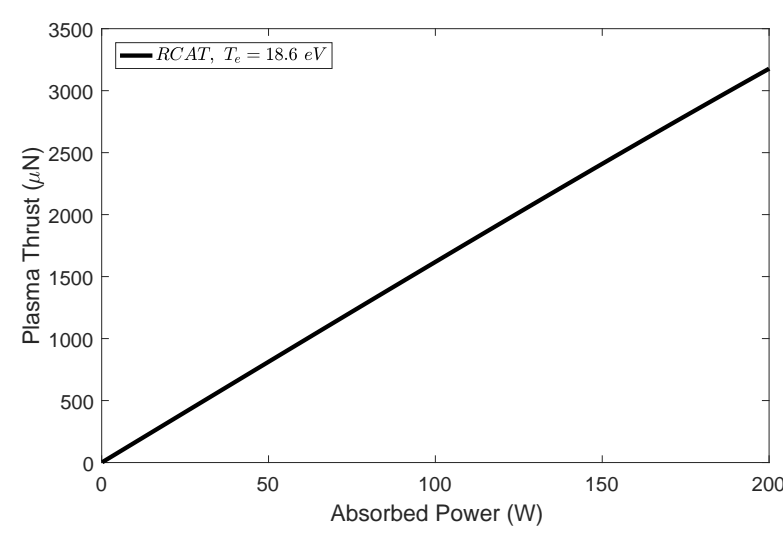

((a))

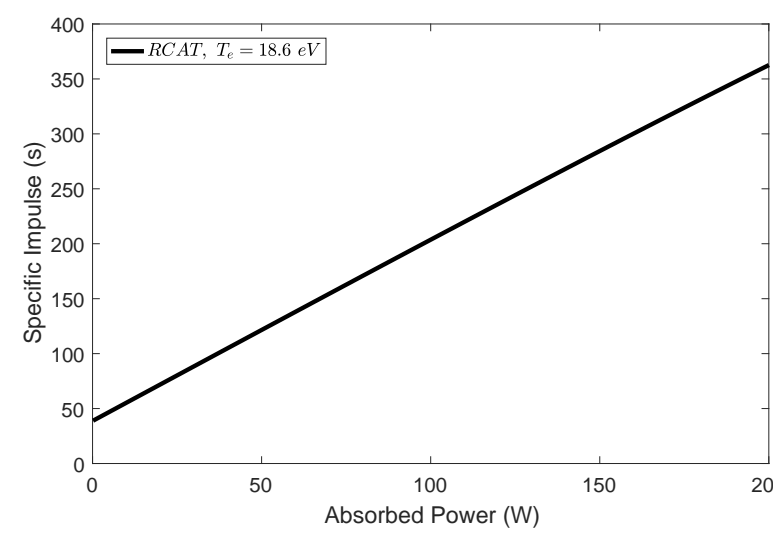

((c))



$((\mathrm{h}))$

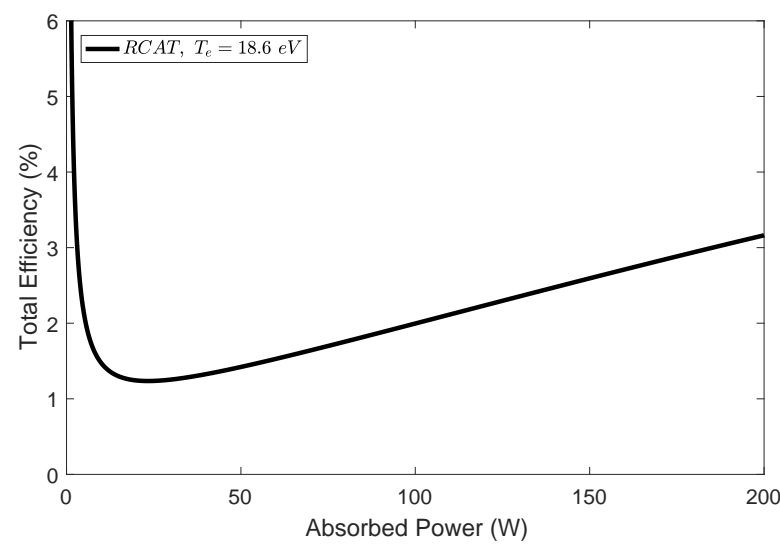

((d))

Figure 16: The (a) plasma thrust, (b) the ratio of the plasma thrust and cold gas thrust, (c) the specific impulse, and (d) total efficiency performance of the revised CAT configuration over an extended power range.

\section{Viability of Magnetic Nozzles for Small Satellites}

To investigate the performance scaling with power the modeled power envelope can simply be extended. The range is extended to $200 \mathrm{~W}$, which can be obtained by a $12 \mathrm{U}$ CubeSat on orbit. As observed in Figure 16 extending the power results in further improvements to thrust, the component of thrust due to the plasma, and specific impulse. The higher power operation also allows for recovery of higher total efficiency, due to improvement in the ratio of the contribution of the plasma and cold gas terms to total thrust. The specific impulse and the total efficiency compare poorly to a state of the art Hall Effect Thruster (HET) performance targets of several 1000 seconds and $\sim 60 \%$, respectively, ${ }^{41}$ but offer significant improvement over the small satellite heritage cold gas systems.

Interestingly, the modified model can explore higher power ranges until it overlaps with the original Lafleur model $^{1}$ (when magnetized ions become important) to explore the performance limitations of magnetic nozzle devices. The state of the art HET typically operates in the $\mathrm{kW}$ range, ${ }^{41}$ so it is possible to compare the performance of magnetic nozzle thruster to the state of the art performance benchmarks at comparable power levels. As reported by Takahashi, et. al. the total efficiency of a magnetic nozzle thruster with a $6.5 \mathrm{~cm}$ diameter by $20 \mathrm{~cm}$ long source region with a magnetic field strength of $100 \mathrm{~s}$ of $\mathrm{G}$ is $8 \%$ at $2 \mathrm{~kW}$ operation. ${ }^{33}$ This does not compare favorably to the $\sim 60 \%$ efficiency reported for HETs, suggesting that performance is not the primary advantage of magnetic nozzle thrusters. Rather, the main advantage is the ability to use corrosive propellants while maintaining long lifetime, due to the lack of plasma-wetted electrodes. 


\section{Conclusion}

The quasi-1D model outlined by Lafleur ${ }^{1}$ is modified to handle partially magnetized ions and is used to determine the critical parameters impacting magnetic nozzle thruster performance. After modification of the detachment criterion the model matches the thrust performance within errorbars of the previously presented configuration of CAT. ${ }^{2}$ The thrust was indirectly measured using a momentum target affixed to the null-type torsional balance thrust stand and the artificial increase in the thrust due to RF pickup is removed. This allows the modified algorithm to be used to explore the effect of plasma density profile, detachment Mach number, the neutral propellant flow rate, and the source aspect ratio on the thruster performance for small thrusters. From these cases it is clear that it is critical to increase the electron temperature to achieve decent performance, either through limiting radial wall losses by limiting the $R_{s} / L_{s}$ ratio or obtaining

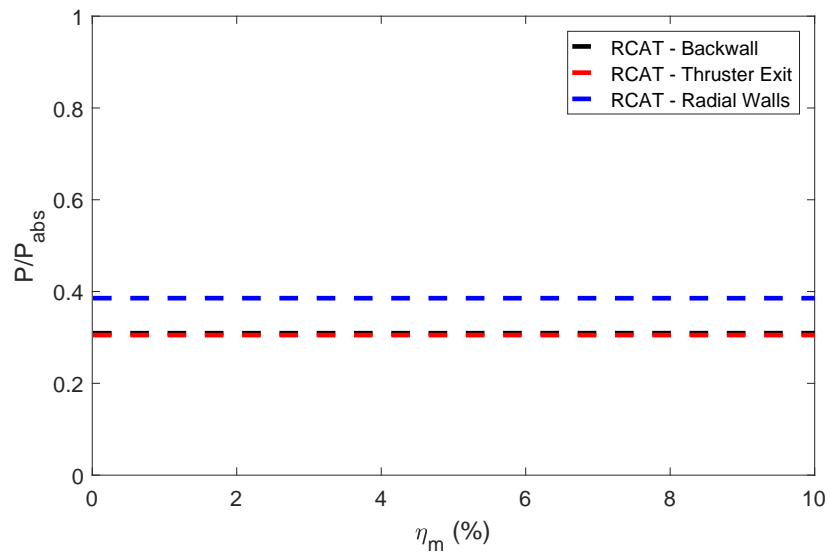

Figure 14: The power loss to the three source boundaries for the recommended CAT configuration. Note that the thruster exit and the backwall nearly overlap. steady operation at lower propellant flow rates while maintaining the absorbed power. Moderate improvement can be also obtained by increasing the detachment Mach number. Within the $50 \mathrm{~W}$ operating envelope the efficiency performance is limited to a few percent due to excessive radial losses and low momentum leaving the source region.

In the configuration reported ${ }^{2}$ the CAT performance did not compare favorably to cold gas for the required operating power, due to poor source aspect ratio and nominal mass flow rate. Changes to the thruster design and operating conditions are discussed to improve the performance, primarily by improving the aspect ratio and adjusting the operating point to a lower propellant flow rate. For small magnetic nozzle thrusters in general the aspect ratio should be maximized and the propellant flow rate minimized, while considering the physical satellite and steady operation constraints. After incorporating improvements to the CAT design improved performance is predicted, but the specific impulse and total efficiency remain low. Using the model it is demonstrated that low power operation limits these two performance metrics to low values, $<200 \mathrm{~s}$ and $<2 \%$, respectively. The performance of higher power, larger magnetic nozzle devices do not compare favorably to state of the art HETs. This suggests that the main advantage of magnetic nozzle thrusters is not performance, but rather the ability to use corrosive propellants without degrading thruster lifetime.

\section{Acknowledgements}

This material is based upon work supported by the National Science Foundation Graduate Research Fellowship Program under Grant No. DGE 1256260, the National Aeronautics and Space Administration (NASA) under Award No. NNX14AD71G, and the Defense Advanced Research Projects Agency (DARPA) under NASA contract Number NNA15BA42C. The authors would like to thank Phase Four, Inc. for their assistance. Thank you to the members of the Plasmadynamics and Electric Propulsion Laboratory for their insightful discussion concerning this research.

\section{References}

${ }^{1}$ Lafleur, T., "Helicon plasma thruster discharge model," Physics of Plasmas, Vol. 21, No. 4, 2014, pp. 043507.

${ }^{2}$ Collard, T. and Sheehan, J. P., Preliminary Measurements of an Integrated Prototype of the CubeSat Ambipolar Thruster, AIAA Propulsion and Energy Forum, American Institute of Aeronautics and Astronautics, 2016, doi:10.2514/6.2016-5042.

${ }^{3}$ Little, J. M., Performance Scaling of Magnetic Nozzles for Electric Propulsion, Ph.D. thesis, 2015.

${ }^{4}$ Rhee, M., Zakrzwski, C., and Thomas, M., "Highlights of Nanosatellite Propulsion Development Program at NASAGoddard Space Flight Center," Small Satellite Conference.

${ }^{5}$ Schmuland, D., Masse, R., and Sota, C., "Hydrazine propulsion module for cubesats," Small Satellite Conference. 
${ }^{6}$ Schmuland, D. T., Carpenter, C., and Masse, R. K., "Mission Applications of the MRS-142 CubeSat High-Impulse Adaptable Monopropellant Propulsion System (CHAMPS)," AIAA Paper, , No. 2012-4269, 2012.

${ }^{7}$ Zondervan, K., Fuller, J., Rowen, D., Hardy, B., Kobel, C., Chen, S.-H., Morrison, P., Smith, T., and Kremer, A., "CubeSat Solid Rocket Motor Propulsion Systems providing Delta-Vs greater than $500 \mathrm{~m} / \mathrm{s}$," Small Satellite Conference.

${ }^{8}$ Bertino-Reibstein, A. and Wuerl, A., "Development of a Warm-Gas Butane System for Microsatellite Propulsion," Small Satellite Conference, 2013.

${ }^{9}$ Dinardi, A. and Persson, M., "High performance green propulsion (HPGP): a flight-proven capability and cost gamechanger for small and secondary satellites," Small Satellite Conference.

${ }^{10}$ Dyer, J., Dinardi, A., and Anflo, K., "First Implementation of High Performance Green Propulsion in a Constellation of Small Satellites," Small Satellite Conference.

${ }^{11}$ Morris, D. and Noble, R., "CubeSat advanced technology propulsion system concept," Small Satellite Conference.

${ }^{12}$ Orr, N., Eyer, J., Larouche, B., and Zee, R., "Precision formation flight: the CanX-4 and CanX-5 dual nanosatellite mission," Small Satellite Conference.

${ }^{13}$ Platt, D., "A monopropellant milli-Newton thruster system for attitude control of nanosatellites," Small Satellite Conference.

${ }^{14}$ Zhuang, T., Shashurin, A., Beilis, I., and Keidar, M., "Ion velocities in a micro-cathode arc thruster," Physics of Plasmas (1994-present), Vol. 19, No. 6, 2012, pp. 063501.

${ }^{15}$ Trezzolani, F., Fabris, A. L., Pavarin, D., Selmo, A., and Manente, M., "Low Power Radio-Frequency Plasma Thruster Development and Testing," 33rd International Electric Propulsion Conference.

${ }^{16}$ Spence, D., Ehrbar, E., Rosenblad, N., Demmons, N., Roy, T., Hoffman, S., Williams, D., Hruby, V., and Tocci, C., "Electrospray propulsion systems for small satellites," Small Satellite Conference.

${ }^{17}$ Shashurin, A., Keidar, M., and Zhuang, T., "Comparative analysis of micro-cathode arc thruster performance," 33rd International Electric Propulsion Conference.

${ }^{18}$ Martel, F., Perna, L., and Lozano, P., "Miniature Ion Electrospray Thrusters and Performance Test on CubeSats," Small Satellite Conference.

${ }^{19}$ Kronhaus, I., Schilling, K., Jayakumar, S., Kramer, A., Pietzka, M., and Schein, J., "Design of the UWE-4 Picosatellite Orbit Control System using Vacuum-Arc-Thrusters," Proceedings of the 33rd International Electric Propulsion Conference, pp. 2013-195.

${ }^{20}$ Ketsdever, A. D., Lee, R. H., and Lilly, T. C., "Performance testing of a microfabricated propulsion system for nanosatellite applications," Journal of Micromechanics and Microengineering, Vol. 15, No. 12, 2005, pp. 2254.

${ }^{21}$ Keidar, M., Haque, S., Zhuang, T., Shashurin, A., Chiu, D., Teel, G., Agasid, E., Tintore, O., and Uribe, E., "Microcathode arc thruster for PhoneSat propulsion," Small Satellite Conference.

${ }^{22}$ Fuchikami, S., Nakamoto, M., Toyoda, K., and Cho, M., "Development of Vacuum Arc Thruster for Nano-Satellite," 33rd International Electric Propulsion Conference, Washington DC, USA, 2013.

$\checkmark{ }^{23}$ Coletti, M., Guarducci, F., and Gabriel, S., "A micro PPT for Cubesat application: Design and preliminary experimental results," Acta Astronautica, Vol. 69, No. 3, 2011, pp. 200-208.

${ }^{24}$ Coletti, M., Ciaralli, S., and Gabriel, S. B., "PPT Development for Nanosatellite Applications: Experimental Results," Plasma Science, IEEE Transactions on, Vol. 43, No. 1, 2015, pp. 218-225.

${ }^{25}$ Ciaralli, S., Coletti, M., and Gabriel, S., "Performance and lifetime testing of a pulsed plasma thruster for Cubesat applications," Aerospace Science and Technology, 2015.

${ }^{26}$ Sheehan, J. P., Timothy, C., Benjamin, W. L., and Ingrid, R., New Low-Power Plasma Thruster for Nanosatellites, Propulsion and Energy Forum, American Institute of Aeronautics and Astronautics, 2014, doi:10.2514/6.2014-3914.

$\checkmark{ }^{27}$ Kuninaka, H. and Satori, S., "Development and Demonstration of a Cathodeless Electron Cyclotron Resonance Ion Thruster," Journal of Propulsion and Power, Vol. 14, No. 6, 1998, pp. 1022-1026.

${ }^{28}$ Williams, L. T. and Walker, M. L., "Thrust measurements of a radio frequency plasma source," Journal of Propulsion and Power, Vol. 29, No. 3, 2013, pp. 520-527.

${ }^{29}$ Williams, L. T. and Walker, M. L., "Thrust Measurements of a Helicon Plasma Source," AIAA/ASME/SAE/ASEE Joint Propulsion Conference Exhibit.

${ }^{30}$ Ling, J., West, M., Lafleur, T., Charles, C., and Boswell, R., "Thrust measurements in a low-magnetic field high-density mode in the helicon double layer thruster," Journal of Physics D: Applied Physics, Vol. 43, No. 30, 2010, pp. 305203.

\$11 Takahashi, K., Lafleur, T., Charles, C., Alexander, P., Boswell, R., Perren, M., Laine, R., Pottinger, S., Lappas, V., and Harle, T., "Direct thrust measurement of a permanent magnet helicon double layer thruster," Applied Physics Letters, Vol. 98, No. 14, 2011, pp. 141503.

${ }^{32}$ Fruchtman, A., Takahashi, K., Charles, C., and Boswell, R., "A magnetic nozzle calculation of the force on a plasma," Physics of Plasmas (1994-present), Vol. 19, No. 3, 2012, pp. 033507.

${ }^{33}$ Kazunori, T., Christine, C., Rod, B., and Akira, A., "Performance improvement of a permanent magnet helicon plasma thruster," Journal of Physics D: Applied Physics, Vol. 46, No. 35, 2013, pp. 352001.

$\checkmark{ }^{34}$ Lafleur, T., Cannat, F., Jarrige, J., Elias, P., and Packan, D., "Electron dynamics and ion acceleration in expandingplasma thrusters," Plasma Sources Science and Technology, Vol. 24, No. 6, 2015, pp. 065013.

${ }^{35}$ Ahedo, E. and Merino, M., "Two-dimensional supersonic plasma acceleration in a magnetic nozzle," Physics of Plasmas (1994-present), Vol. 17, No. 7, 2010, pp. 073501.

${ }^{36}$ Ahedo, E. and Merino, M., "On plasma detachment in propulsive magnetic nozzles," Physics of Plasmas (1994-present), Vol. 18, No. 5, 2011, pp. 053504.

${ }^{37}$ Ahedo, E. and Merino, M., "Two-dimensional plasma expansion in a magnetic nozzle: Separation due to electron inertia," Physics of Plasmas, Vol. 19, No. 8, 2012, pp. 083501. 
\$38Hooper, E., "Plasma detachment from a magnetic nozzle," Journal of Propulsion and Power, Vol. 9, No. 5, 1993, pp. $757-763$.

39 Mario, M. and Eduardo, A., "Plasma detachment in a propulsive magnetic nozzle via ion demagnetization," Plasma Sources Science and Technology, Vol. 23, No. 3, 2014, pp. 032001.

${ }^{40}$ Simpson, J. C., Lane, J. E., Immer, C. D., and Youngquist, R. C., "Simple analytic expressions for the magnetic field of a circular current loop," 2001.

${ }^{41}$ Scott J., H., Sarah E., C., and Alec D., G., "30-kW Performance of a 100-kW Class Nested-Channel Hall Thruster," . 